\title{
Outbreak of acute hemorrhagic conjunctivitis in Yunnan, People's Republic of China, 2007
}

\author{
Dongmei Yan', Shuangli Zhu', Yong Zhang1', Jing Zhang², Yongming Zhou ${ }^{3}$ and Wenbo Xu*1
}

\begin{abstract}
An outbreak of acute hemorrhagic conjunctivitis (AHC) occurred in Yunnan Province, China between August and September in 2007. A total of 3,597 cases were officially reported and the incidence rate reached 1390.94/100,000. Descriptive epidemiological analysis of the outbreak was conducted using the data from National Disease Supervision Information Management System (NDSIMS). To determine the causative agent for this outbreak and to analyze their genetic features, 30 conjunctival swabs and 19 paired serum specimens of acute and convalescent phase were collected from 30 patients with AHC, and viral isolation, molecular typing, antibody assay and phylogenetic analysis were performed.

11 virus strains were isolated from 30 conjunctival swabs. Amplification and sequencing of the VP4 region of these strains identified that coxsackievirus A24 variant (CA24v) could be the causative agent of the AHC outbreak and this was further confirmed by subsequent virus neutralizing antibody test on 19 paired serum specimens. Phylogenetic analysis based on the $3 \mathrm{C}$ regions showed that the Yunnan CA24v strains belonged to Group 3 and clustered with the strains isolated from worldwide AHC outbreaks after 2002. Phylogenetic analysis based on the partial VP1 revealed that the Yunnan strains differed from the strains isolated from AHC outbreak in Guangdong of China in 2007 with 2.8 - 3.0\% nucleotide divergence, suggesting that two different lineages of CA24v caused the independent AHC outbreaks in Yunnan and Guangdong, respectively.
\end{abstract}

\section{Findings}

Acute hemorrhagic conjunctivitis (AHC) is a highly contagious epidemic disease, commonly caused by Enterovirus 70 and Coxsackievirus A24 variant (CA24v). CA24v is an antigenic variant of the CA24 strain and was first isolated from an outbreak of AHC in Singapore in 1970 [1]. The AHC outbreaks caused by CA24v were restricted to Southeast Asia and India until 1985, and then spread rapidly to other regions of the world [2-4].

In China, the first outbreak of AHC was reported in 1971 [5]. Since then, outbreaks of AHC have been regularly reported [6,7]. Notifiable infectious diseases in China are classified into Class A, B, and C according to the degree of risk from the highest to the lowest. AHC belongs to Class $\mathrm{C}$ notifiable infectious disease. When a case is diagnosed as AHC, physicians are required to reg-

\footnotetext{
* Correspondence: wenbo_xu1@yahoo.com.cn
}

1 WHO WPRO Regional Reference Poliomyelitis Laboratory and State Key Laboratory for Molecular Virology \& Genetic Engineering, National Institute for Viral Disease Control and Prevention, Chinese Center for Disease Control and Prevention, 27 Nanwei Road, Beijing 100050, China

Full list of author information is available at the end of the article ister the case of AHC to the National Disease Supervision Information Management System (NDSIMS), which is a direct reporting network system for notifiable infectious diseases in China. According to NDSIMS, a total of 74,263 AHC cases were reported in China in 2007, representing an increase of $540.58 \%$ compared with 11,593 cases in 2006. There were 134 reported outbreaks, spreading to 14 provinces and affecting 10,165 people. Other 64098 are sporadic cases. These data showed that a nationwide AHC epidemic occurred in China in 2007.

An outbreak of AHC occurred in Suijiang and its neighboring Shuifu Counties, Yunnan Province between August and September in 2007. In this study, descriptive epidemiological analysis was conducted using the data from NDSIMS and the causative agent was determined. 30 conjunctival swabs and 19 paired of acute and convalescent serum specimens were collected from 30 patients with AHC in Suijiang and Shuifu in September, 2007(Table 1). Two hundred microliter of the swab specimen was inoculated onto RD (rhabdomyosarcoma) and HEp-2 cells (human epidermoid carcinoma of the larynx). The cells were observed for 7 days for cytopathic effect 





Table 1: Epidemiological information and laboratory results of acute hemorrhagic conjunctivitis in Yunnan, China, 2007 (Continued)

\begin{tabular}{|c|c|c|c|c|c|c|c|c|c|c|c|c|}
\hline SF15 & $16 / \mathrm{M}$ & Student & 8 Sep. 2007 & 10 Sep. 2007 & 10 Sep. 2007 & 1 & $\mathrm{Neg}$ & $\mathrm{Neg}$ & 1 & 1 & I & $\mathrm{Neg}$ \\
\hline SF16 & $18 / \mathrm{M}$ & Student & 9 Sep. 2007 & 10 Sep. 2007 & 10 Sep. 2007 & I & Neg & Neg & / & / & I & $\mathrm{Neg}$ \\
\hline SF17 & $16 / \mathrm{M}$ & Student & 9 Sep. 2007 & 10 Sep. 2007 & 10 Sep. 2007 & / & Neg & Pos & I & I & I & Pos \\
\hline SF18 & $18 / \mathrm{M}$ & Student & 9 Sep. 2007 & 10 Sep. 2007 & 10 Sep. 2007 & I & Neg & Pos & / & / & / & Pos \\
\hline SF19 & $17 / M$ & Student & 8 Sep. 2007 & 10 Sep. 2007 & 10 Sep. 2007 & / & Neg & Neg & / & / & / & $\mathrm{Neg}$ \\
\hline SF20 & $17 / \mathrm{M}$ & Student & 9 Sep. 2007 & 10 Sep. 2007 & 10 Sep. 2007 & / & $\mathrm{Neg}$ & $\mathrm{Neg}$ & / & / & l & $\mathrm{Neg}$ \\
\hline
\end{tabular}


(CPE). Two blind passages were performed when no CPE was observed.

Viral RNA was extracted from virus culture supernatant with a QIAamp Mini Viral RNA Extraction kit (Qiagen, Valencia, CA, USA). RT-PCR was performed using the primers OL68-1 and MD91 targeted for the VP4 region of the human enterovirus as described previously [8]. The obtained PCR products were purified with a QIAquick PCR Purification Kit (Qiagen, kk, Japan). The nucleotide sequence was determined by ABI PRISM 3100 Genetic Analyzer (Applied Biosystems, Hitachi, Japan) using BigDye Terminator Version 3.0 (Applied Biosystems, CA, USA). The serotype was determined by comparing the VP4 nucleotide sequence with those available at GenBank database using the BLAST program http:// blast.ncbi.nlm.nih.gov/Blast.cgi from NCBI according to the criteria for molecular typing proposed previously [8].

The neutralizing antibody titers of the 19 paired serum specimens were determined by a virus neutralization assay. In brief, serial dilutions of the sera were mixed with $10050 \%$ cell culture infective doses (CCID50) per $50 \mathrm{ul}$ of the CA24v virus [Strain SF3, isolated from this outbreak, Genbank number EU596581 (VP1 region) and GQ229400(3C region)], incubated at $36^{\circ} \mathrm{C}$ for 2 hours and inoculated onto HEp-2 cells. A 4-fold or greater rise of CA24v neutralizing antibodies titre between acute and convalescent serum indicated an acute infection of CA24v.
For the molecular epidemiologic study, the partial VP1 and $3 \mathrm{C}$ region of Yunnan strains were amplified and sequenced with primers that flanked the VP1 region (forward, CVA24v-2407S: 5'-GTGAGTGCTTGCCCAGATTT-3'; reverse, CVA24v-3438A: 5'ATACACCGCCATGTTCTGGT-3') designed in this study and primers $3 \mathrm{C} 1 / 3 \mathrm{C} 2$ that flanked the entire $3 \mathrm{C}$ region [9]. The sequences of partial VP1 and $3 \mathrm{C}$ regions of the Yunnan isolates were aligned with those of the corresponding region of the different geographic and temporal CA24v strains using the CLUSTAL W method [10] in MEGA 4.0 [11]. Phylogenetic trees were constructed using the neighbor-joining method in MEGA 4.0 with bootstrap analysis performed on 1000 replicates. All sequences reported in this study were deposited into GenBank under accession number EU596577 to EU596589 and GQ229391 to GQ229410.

Shuifu and Suijiang Counties are adjacent counties located in the northeast of Yunnan Province and consist of 8 townships with a total population of 258,602 people. This AHC epidemic affected all 8 townships. By September 30,2007 , a total of 3,597 cases were officially reported by NDSIMS. The incidence rate reached 1390.94/ 100,000 , no death and severe case occurred. Daily distribution of acute hemorrhagic conjunctivitis cases between August and September was shown in Figure 1.The first clinical diagnosed patient of the outbreak was identified on August 8, 2007 in Suijiang. Subsequently, the number

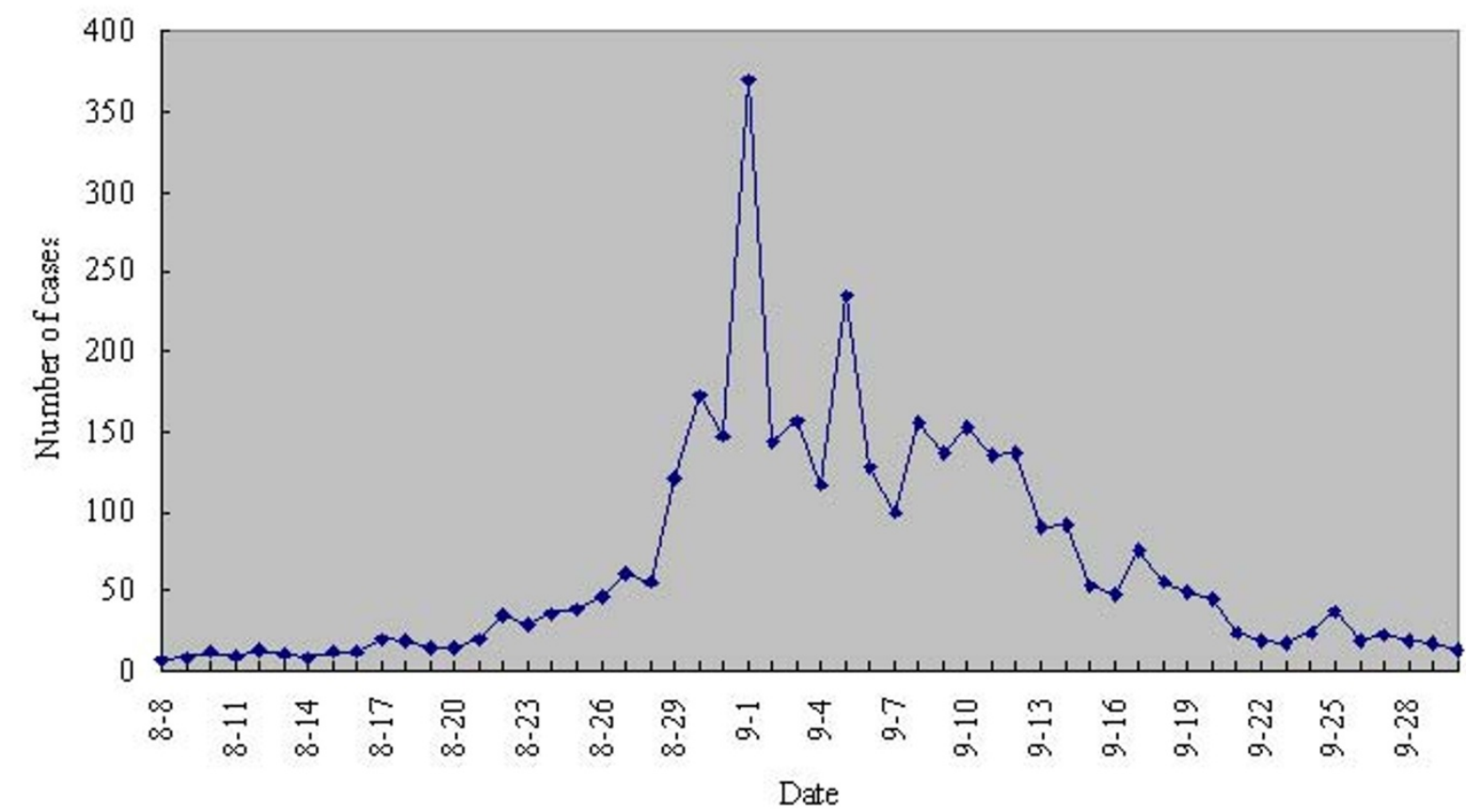

Figure 1 Daily distribution of acute hemorrhagic conjunctivitis cases in Suijiang and Shuifu Counties, Yunnan in 2007. 


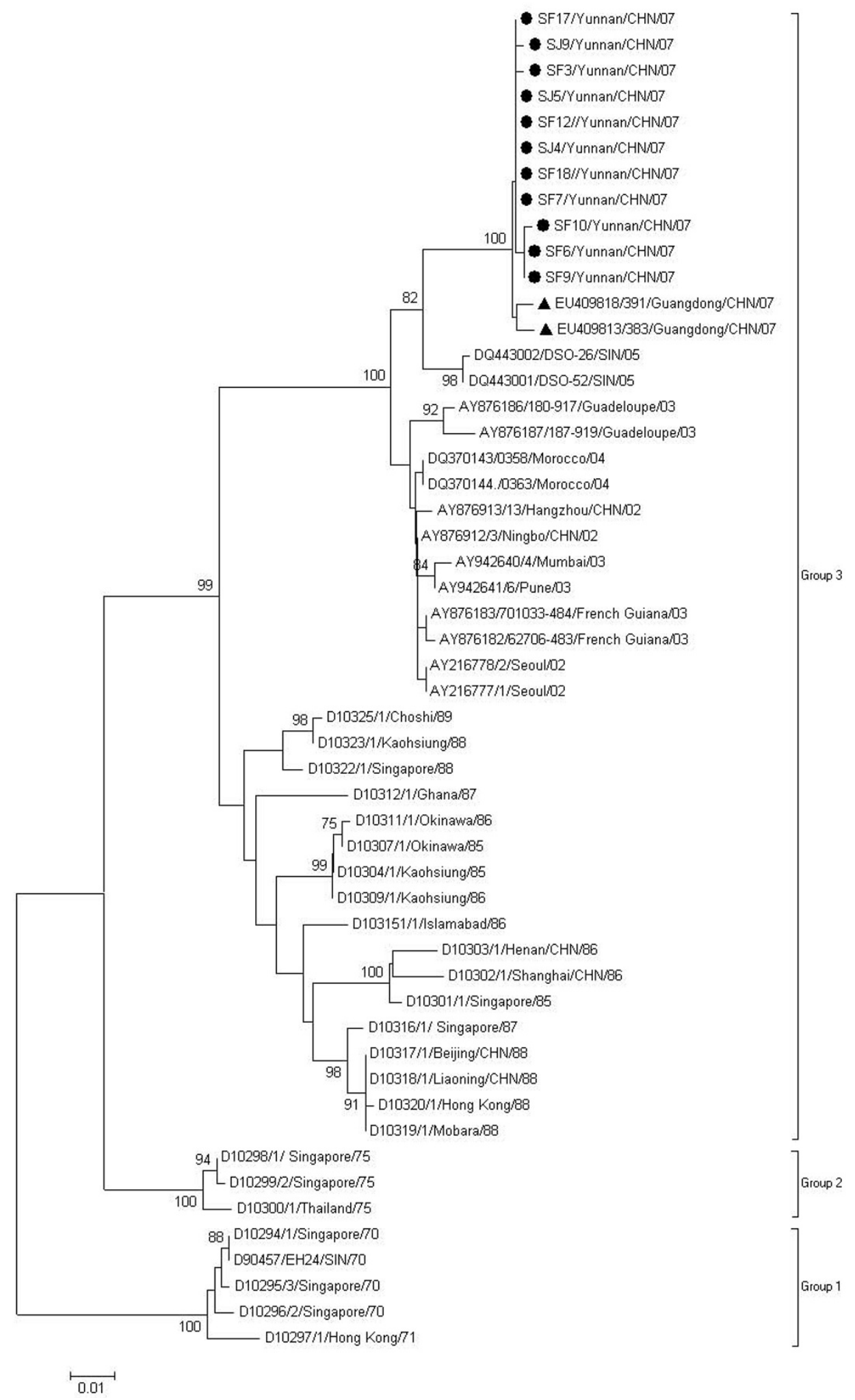

Figure 2 Phylogenetic analysis based on the $3 \mathrm{C}$ region. The nucleotide sequences of $3 \mathrm{C}$ region of Yunnan strains were compared with those of the CA24v strains from AHC outbreaks worldwide. In the unrooted tree obtained, Yunnan strains are indicated by black spots. Guangdong isolates were indicated by black triangle. The bootstrap values of at least 75\% are shown at tree nodes. Every strain is indicated by GenBank accession number, strain code, country/region followed by year of isolation 


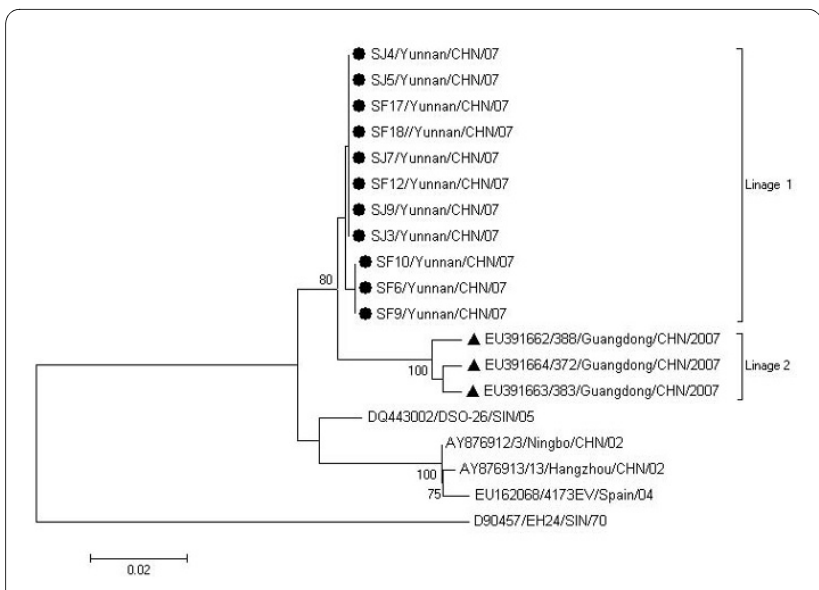

Figure 3 Phylogenetic analysis based on the partial VP1 region The nucleotide sequences of partial VP1 region of Yunnan strains were compared with those of Guangdong strains and other CA24v strains with entire VP1 nucleotide sequence available in GenBank. The prototype CA24v strain EH24/70 was used as the root. Yunnan strains are indicated by black spots. Guangdong isolates were indicated by black triangle. The bootstrap values of at least 75\% are shown at tree nodes. Every strain is indicated by GenBank accession number, strain code, country/region followed by year of isolation.

of cases increased, reached a peak in the beginning of September and returned to baseline in late September (Figure 1). The age of the patients ranged from 3months to 84 years. The ratio between male and female was 1.71:1. Among all the patients, $46.4 \%$ were farmers, followed by students (31.9\%) and the mean age was 37.7 years for farmers and 15.2 for students. The most common symptoms were conjunctival congestion (100\%), followed by ocular stabbing pain (73.2\%) and photophobia (64.6\%). Some patients also appeared systemic symptoms such as fever (2.5\%), fatigue (2.7\%) and limbs pain (1.7\%). 91.3\% of all patients had a clear history of contact with people with similar symptoms.

As shown in table 1, HEp-2 cell was the only cell line capable of CA24v isolation in this study. Virus were isolated from 11(37\%) of 30 swabs and VP4 regions were successfully amplified and sequenced for all 11 isolates (Table 1). All the sequences obtained have the highest identity (99.0-100.0\%) with the CA24v strains (GenBank No. EU221286 to EU221294) isolated between July and October, 2007 in Guangdong Province of China [12], suggesting that CA24v could be the causative agent of the AHC outbreak. The virus neutralizing antibody analysis for 17 of 19 paired serum specimens showed a 4 fold or greater rise in neutralizing antibodies titer which provided further evidence that CA24v was the causative agent of the AHC outbreak in Yunnan (Table 1).

Similar to the previous studies $[12,13]$, the phylogenetic analysis based on the $3 \mathrm{C}$ region revealed that all the $\mathrm{CA} 24 \mathrm{v}$ strains were divided into three major groups.
They are Group1 containing CA24v isolated from 1970 to 1971; Group 2, from 1975; Group 3, from 1985 to 2007. The Yunnan strains belonged to Group 3 and clustered with the strains isolated from worldwide AHC outbreaks after 2002 (Figure 2). The Yunnan strains were most closely related to those isolated in 2007 in Guangdong Province of China [12] with high nucleotide identities of $99.1-99.3 \%$ in $3 \mathrm{C}$ (Figure 2). In contrast to $3 \mathrm{C}$ region, the phylogenetic tree based on the partial VP1 region of CA24v strains showed more divergence between Yunnan and Guangdong strains, which clearly belonged to two different lineages (Lineage 1 and Lineage 2), respectively (Figure 3). The nucleotide identities within Lineages 1 and Lineages 2 were $99.7-100 \%$ and $98.6-99.2 \%$ respectively and A $2.8-3.0 \%$ nucleotide divergence was found between these two lineages.

The outbreak of AHC described in this study occurred between August and September in Yunnan Province. The climate of this period in Yunnan is characterized by high temperatures and frequent rainfall, which may be two climatic factors involved in the rapid spread of CA24v strains. In this outbreak, the most patients were farmers (46.4\%), while workers $(37.56 \%)$ were the most predominant patient in the contemporaneous AHC outbreak in Guangdong [12]. That may be because farmers are the main population (accounting for $78 \%$ of the total number) in Shuifu and Shuijiang Counties of Yunnan. Moreover, August and September are in the rice harvest season and local farmers have the habit of laboring together, resulting in exposure time and frequency increased significantly. In addition, in rural areas such as these two counties, the phenomenon of sharing the basins and towels was very common which played an important pole in causing the outbreak.

Our study demonstrated that CA24v was the causative agent of this AHC outbreak in Yunnan, southern China. Moreover, AHC outbreaks reported in Guangdong (southern China) [12] and Beijing (northern China) [14] in 2007, were also caused by CA24v. Although the information on the causative agent of AHC outbreaks in other provinces was not obtained, it is possible that CA24v was major causative agent during the national AHC outbreak considering the close temporal and geographical relation.

Although $3 \mathrm{C}$ region was commonly used for molecular epidemiological analysis of CVA24v strains, recent studies have demonstrated that capsid encoding region may be more informative than the $3 \mathrm{C}$ region because of the high recombination rate in nonstructural region between enteroviruses and serological pressure on the capsid encoding region which therefore may evolve faster than the region encoding for the nonstructural proteins. In this study, compared with $3 \mathrm{C}$ region, phylogenetic tree based on the partial VP1 region of CA24v strains showed more divergence between Yunnan and Guangdong 
strains and clearly differentiated Yunnan lineage and Guangdong linage, indicating that two independent outbreaks occurred in Yuannan and Guangdong although the AHC outbreaks in these two provinces was temporally and geographically related.

\section{Abbreviations}

AHC: acute hemorrhagic conjunctivitis; CA24v: coxsackievirus A24 variant; RTPCR: Reverse transcription-polymerase chain reaction.

\section{Competing interests}

The authors declare that they have no competing interests.

\section{Authors' contributions}

Dongmei Yan and Shuangli Zhu performed the experiment. Jing Zhang and Yong-ming Zhou had made substantial contributions to acquisition of epidemiological information. Dongmei Yan drafted the manuscript. Yong Zhang and Wenbo Xu revised the manuscript. All authors read and approved the final manuscript.

\section{Acknowledgements}

We thank all personnel from Suijiang Center for Disease Control and Prevention and Shuifu Center for Disease Control and Prevention, Zhaotong, People's Republic of China for the specimen collection.

This work was supported by Grant (National Science and Technology Support Program 2008BAI56B01 and National Infectious Disease Surveillance Program: 2009ZX10602) from the Ministry of Health, People's Republic of China.

\section{Author Details}

'WHO WPRO Regional Reference Poliomyelitis Laboratory and State Key Laboratory for Molecular Virology \& Genetic Engineering, National Institute for Viral Disease Control and Prevention, Chinese Center for Disease Control and Prevention, 27 Nanwei Road, Beijing 100050, China, ${ }^{2}$ Office for Disease Control and Emergency Response, Chinese Center for Disease Control and Prevention, 27 Nanwei Road, Beijing 100050, China and 3Yunnan Center for Disease Control and Prevention, Dongshi Road, Kunming 650022, China

Received: 16 March 2010 Accepted: 25 June 2010

Published: 25 June 2010

\section{References}

1. Lim KH, Yin-Murphy M: An epidemic of conjunctivitis in Singapore in 1970. Singapore Med J 1971, 12:247-249

2. Miyamura K, Yamashita K, Takeda N, Ogino T, Utagawa E, Yamazaki S, Fukumura K, Uehara T, Shinjo N: The first epidemic of acute hemorrhagic conjunctivitis due to a coxsackievirus A24 variant in Okinawa, Japan, in 1985-1986. Jpn J Med Sci Biol 1988, 41:159-174.

3. Sawyer LA, Hershow RC, Pallansch MA, Fishbein DB, Pinsky PF, Broerman SF, Grimm BB, Anderson LJ, Hall DB, Schonberger LB: An epidemic of acute hemorrhagic conjunctivitis in American Samoa caused by coxsackievirus A24 variant. Am J Epidemiol 1989, 130:1187-1198.

4. Tavares FN, Costa EV, Oliveira SS, Nicolai CC, Baran M, da Silva EE: Acute hemorrhagic conjunctivitis and coxsackievirus A24v, Rio de Janeiro, Brazil, 2004. Emerg Infect Dis 2006, 12:495-497.

5. Mu GF: An etiological study of acute hemorrhagic conjunctivitis in Beijing area in 1984 [in Chinese]. Zhonghua Yan Ke Za Zhi 1989, 25:20-22.

6. Meng R, Qiu Y, Yan L: Study on etiology of acute hemorrhagic conjunctivitis in Qingdao during 1997 [in Chinese]. Zhonghua Shi Yan He Lin Chuang Bing Du Xue Za Zhi 1999, 13:186-187.

7. Mu GF: Etiology of 1988 epidemic of acute hemorrhagic conjunctivitis in Beijing [in Chinese]. Zhonghua Yu Fang Yi Xue Za Zhi 1990, 24:129-131.

8. Ishiko H, Shimada Y, Yonaha M, Hashimoto O, Hayashi A, Sakae K, Takeda $\mathrm{N}$ : Molecular diagnosis of human enteroviruses by phylogeny-based classification by use of the VP4 sequence. J Infect Dis 2002, 185:744-754.

9. Ishiko H, Takeda N, Miyamura K, Kato N, Tanimura M, Lin KH, Yin-Murphy M, Tam JS, Mu GF, Yamazaki S: Phylogenetic analysis of a coxsackievirus A24 variant: the most recent worldwide pandemic was caused by progenies of a virus prevalent around 1981. Virology 1992, 187:748-759.
10. Thompson JD, Higgins DG, Gibson TJ: CLUSTAL W: improving the sensitivity of progressive multiple sequence alignment through sequence weighting, position-specific gap penalties and weight matrix choice. Nucleic Acids Res 1994, 22:4673-4680.

11. Tamura K, Dudley J, Nei M, Kumar S: MEGA4: Molecular Evolutionary Genetics Analysis (MEGA) software version 4.0. Mol Biol Evol 2007, 24:1596-1599

12. Wu D, Ke CW, Mo YL, Sun LM, Li H, Chen QX, Zou LR, Fang L, Huang P, Zhen HY: Multiple outbreaks of acute hemorrhagic conjunctivitis due to a variant of coxsackievirus A24: Guangdong, China, 2007. J Med Virol 2008, 80:1762-1768.

13. Lévêque N, Amine IL, Cartet G, Hammani AB, Khazraji YC, Lina B, Muyembe $\mathrm{JJ}$, Norder $\mathrm{H}$, Chomel JJ: Two outbreaks of acute hemorrhagic conjunctivitis in Africa due to genotype III coxsackievirus A24 variant. Eur J Clin Microbiol Infect Dis 2007, 26:199-202.

14. Ding LX, Zhang Y, Li J, Dou XF, Yan DM, Zhu SL, An HQ, Xu WB: Outbreak of acute hemorrhagic conjunctivitis in Beijing City in 2007 caused by coxsackievirus A24 variant: molecular identification and phylogenetic analysis. Bing Du Xue Bao 2009, 25:251-256.

doi: $10.1186 / 1743-422 X-7-138$

Cite this article as: Yan et al., Outbreak of acute hemorrhagic conjunctivitis in Yunnan, People's Republic of China, 2007 Virology Journal 2010, 7:138

\section{Submit your next manuscript to BioMed Central} and take full advantage of:

- Convenient online submission

- Thorough peer review

- No space constraints or color figure charges

- Immediate publication on acceptance

- Inclusion in PubMed, CAS, Scopus and Google Scholar

- Research which is freely available for redistribution 